Document downloaded from:

http://hdl.handle.net/10251/121077

This paper must be cited as:

Coll, C.; Sánchez, E. (2018). An N-periodic control for coupled systems. International Journal of Systems Science. 49(5):1040-1046.

https://doi.org/10.1080/00207721.2018.1441468

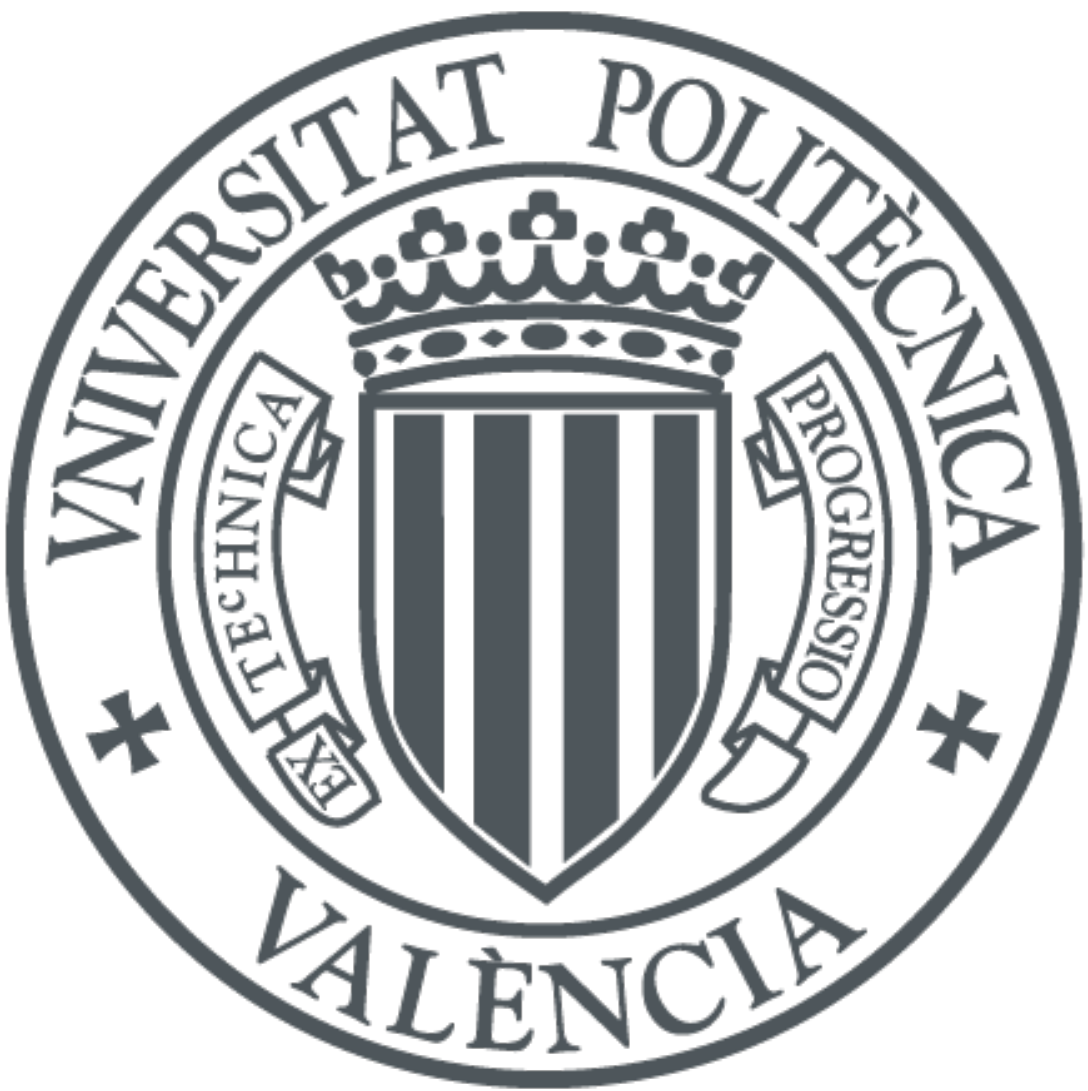

The final publication is available at

http://doi.org/ DOI: 10.1080/00207721.2018.1441468

Copyright Taylor \& Francis

Additional Information 


\title{
An $N$-periodic control for coupled systems
}

\author{
Carmen Coll and Elena Sánchez \\ Instituto de Matemática Multidisciplinar. Universitat Politècnica de València. Camino de \\ Vera, 14. 46022 Valencia. Spain
}

\section{ARTICLE HISTORY}

Compiled February 9, 2018

\begin{abstract}
This paper analyzes the stabilization of two coupled systems described by difference equations via an $N$-periodic feedback control. For this, it is considered a discretetime approach with two compartmental systems, where each system has communication with the other one. An $N$-periodic controller is proposed to attenuate the interconnections affecting to stability of the process. It is designed so in each interval of amplitude $N$, the control is only added at initial time and there is not control in the rest of the time. This feedback control ensures the stability of the closed-loop system. Finally, an example is given to illustrate the theoretical results.
\end{abstract}

\section{KEYWORDS}

Interconnected systems, stability, feedback control, non-negative matrices, discrete-time systems, periodic systems

\section{AMS CLASSIFICATION}

93C55, 93D15, 15XX.

\section{Introduction}

Compartmental models are composed of homogeneous interconnected subsystems (or compartments), i. e. models in which the dynamic units are divided into a set of different groups. It is important to point out the use of dynamic models to predict the future evolution of a real process, in particular, non-negative and compartmental models are applied to several classes of coupled systems in engineering, biology, chemistry, ecology and epidemiology, see Cantó et al. (2014a) and Van den Hof (1998). In particular, non-negative systems are used to model processes involving conservations laws for describing transfer or accumulation between compartments where a variable has an effect on other variables of the model. It is the case, for example, of models involving a network, Li \& Shuai (2010) and Boccaletti et al. (2014) and the references given there. Many of these applications can be modeled by a discrete-time linear system, and a topic of continuing interest is designing control systems that maintain stability, structural properties and performance in presence of constrained signals, Álvarez et al. (2016); Cantó et al. (2014b); Ellis et al. (2014) and Su et al. (2012).

On the other hand, periodic control actions are commonly used in control theory, which leads us to a closed model with an $N$-periodic state matrix. The periodic systems 
are useful for multirate control where the measurement samples and control calculations must be performed at different frequencies. Examples in engineering applications such as electrical circuit networks, aerospace engineering and chemical processing, can be found in Ahn et al. (2010) and Tadeo \& Rami (2010). Furthermore, for nonnegative systems, the condition of positivity of the state variables restricts the kind of control that we can use. Note that, at all times we must ensure that the closed-loop system has non-negative solution.

In this paper, we consider a discrete-time model formed by two interconnected systems, initially independents, but with interactions between them. For example, we consider a network which consists of two compartments interconnected. The usual approach to model such systems is to use graphs whose nodes represent states and the directed links stand for the interactions between them. In our case we use an algebraic approach to model coupled systems. This allows us to use matrix theory techniques and methods to show for new properties in our model.

The connections between the compartments produce perturbations. These disturbances may affect, for instance, stability of the network even from asymptotically stable subsystems. In this case, we need to eliminate these disturbances in order to that the full system is also asymptotically stable. When a large-scale system is considered it is usual to partition the stabilization analysis into tractable sub-problems, for instance, an study of the stability problem for coupled systems is given in Li \& Shuai (2010). As result, the full process is controlled by several independent controllers which all together represent a decentralized controller. Adaptive control and sliding mode techniques are often used when there exist disturbances due to estimation of some parameters, see Mahmoud \& Qureshi (2012); Zhang et al. (2000). The approach developed in this work is not based to uncertainties caused by the presence of unknown parameters. The proposed control scheme is a state feedback applied to all subsystems but on times separated by a periodic amplitude. The designed N-periodic feedback controller is not decentralized controller but it ensures stability and achieves economic efficiency versus an invariant feedback.

To achieve these objectives the feedback is designed so that its action on the coupled systems leads to a periodic disconnection between the subsystems.So, the main contribution of this work is the construction of an $\mathrm{N}$ - periodic feedback that allows us to apply the control action in a single instant to stabilize the model. This is one of the advantages of this control design compared to others, since we only need to act on the coupled systems once every period of time. This makes the feedback problem an economically viable option that involves some benefits in the performance of the closed-loop system. Moreover, we obtain the optimal period such that we can be without acting on the system and it to remain stable. In addition, the designed feedback has advantages over the application of an invariant feedback which acts at all times, specifically, in the case that the application of the feedback is interpreted as a disconnection.

The paper is organized as follows. In the next Section, we formulate the problem and introduce some preliminary results. In Section 3, we design and analyze a feedback control to solve the proposed problem. An example to illustrate the theoretical results obtained in the paper is presented in Section 4 and conclusions are given in Section 5 . 


\section{Problem formulation}

Models from real processes with non-negative states are considered. Note that, the model presented can be applied to any number of compartments, provide that the model structure is maintained. The choice of only two interconnected compartments or systems is due to simplification of some proofs of the results. Mathematically stated, the discrete-time system is described by

$$
x(k+1)=A x(k)+B u(k)
$$

where $x(\cdot)=\left(\begin{array}{c}x_{1}(\cdot) \\ x_{2}(\cdot)\end{array}\right), x_{i}(\cdot) \in \mathbb{R}_{+}^{n}, i=1,2$, and $u(\cdot) \in \mathbb{R}_{+}^{2 n}$, such that the solution of the system is non-negative for all $k \in \mathbb{Z}$. To ensure this property, the matrices of the system must be non-negative, that is, each entry of the matrix must be non-negative with at least one of them positive. In this case, we denote $A \geq 0$ and $B \geq 0$, see Berman \& Plemmons (1994), and the system by $(A, B) \geq 0$.

The network considered is formed by two systems

$$
x_{j}(k+1)=A_{j} x_{j}(k), \quad j=1,2,
$$

where each state influences the following states in both compartments. Then, the state matrix of the full system $x(k+1)=A x(k)$ is given by

$$
A=\left(\begin{array}{cc}
A_{1} & D_{1} \\
D_{2} & A_{2}
\end{array}\right)
$$

where $A_{j}, D_{j}$ are non-negative lower triangular matrices with $\operatorname{diag}\left(A_{j}\right)=\left(a_{j}^{i}\right)_{i=1}^{n}$, $0<a_{j}^{i}<1$ and $\operatorname{diag}\left(D_{j}\right)=\left(d_{j}^{i}\right)_{i=1}^{n}, j=1,2$ with $\operatorname{diag}(\cdot)$ denoting the entries on the diagonal of a matrix. Note that the entries of the matrix $D_{j}, j=1,2$, represent the interactions caused by one subsystem on the other one.

It is known that the stability of a discrete-time linear system is related to the stability of its state matrix. In the positive case, if the interconnected system $x(k+1)=$ $A x(k)$, with $A$ given in (2), is asymptotically stable, then the subsystems $x_{j}(k+1)=$ $A_{j} x_{j}(k), \quad j=1,2$, are asymptotically stable. That is because $\rho\left(\begin{array}{cc}A_{1} & O \\ O & A_{2}\end{array}\right) \leq \rho(A)$ when the matrices are non-negative $(\rho(\cdot)$ denotes the spectral radius of a matrix). But reciprocally, the effect of the disturbances that appears by the coupling between the systems can lead to the destabilization of the full process.

In system (1)-(2) we assume that the subsystems $x_{j}(k+1)=A_{j} x_{j}(k), \quad j=1,2$ are asymptotically stable systems, that is $\rho\left(A_{j}\right)<1, j=1,2$, see Bhatia (1997), but system (1)-(2) is unstable when $u(\cdot) \equiv 0$. If this occurs, the transfer matrix of system (1), $G(z)=(z I-A)^{-1} B$, has at least a pole in modulus greater than or equal to 1 , or equivalently, the matrix $A$ is not stable, $\rho(A) \geq 1$. 


\subsection{About the spectrum of matrix $A$}

Now, we fix our attention in the structure of matrix $A$. By a suitable permutation matrix $P$ we can reorder the rows and columns of the matrix $A$, such that $P A P^{T}=\tilde{A}$ is a lower triangular block matrix which diagonal is given by $\operatorname{diag}(A)=\left(\tilde{A}_{i}\right)_{i=1}^{n}$ where $\tilde{A}_{i}$ is a non-negative matrix with positive diagonal entries given by

$$
\tilde{A}_{i}=\left(\begin{array}{cc}
a_{1}^{i} & d_{1}^{i} \\
d_{2}^{i} & a_{2}^{i}
\end{array}\right), i=1, \ldots, n .
$$

So matrix $\tilde{A}$ is similar to matrix $A$ and both matrices have the same spectrum. From now on $\sigma(\cdot)$ denotes the spectrum of a matrix.

The characteristic polynomial of $A$ is given by $|\lambda I-A|=\prod_{i=1}^{n}\left|\lambda I-\tilde{A}_{i}\right|$. It is clear that $\sigma(A)$ is directly related to $\sigma\left(\tilde{A}_{i}\right)$.

We study $\sigma\left(\tilde{A}_{i}\right)$ in terms of the entries of matrix $\tilde{A}_{i}$. The eigenvalues of matrix $\tilde{A}_{i}$ are $\lambda_{1,2}^{i}=\frac{a_{1}^{i}+a_{2}^{i} \pm \Delta_{i}}{2}$ with $\Delta_{i}=\sqrt{\left(a_{1}^{i}-a_{2}^{i}\right)^{2}+4 d_{1}^{i} d_{2}^{i}} \geq 0$.

For each $i=1,2 \ldots, n$ :

a) $d_{1}^{i} d_{2}^{i}=0$ : If $a_{1}^{i}=a_{2}^{i}$ and we have $\Delta_{i}=0$ and $\lambda_{1}^{i}=\lambda_{2}^{i}=a_{1}^{i}$. If $a_{1}^{i} \neq a_{2}^{i}, \lambda_{1}^{i}=a_{1}^{i}$ and $\lambda_{2}^{i}=a_{2}^{i}$. From $\rho\left(A_{j}\right)<1$ we have $\lambda_{j}^{i}<1, j=1,2$.

b) $d_{1}^{i} d_{2}^{i} \neq 0$ : We have $\Delta_{i} \neq 0$ and $\lambda_{1}^{i}>\lambda_{2}^{i}$. Moreover if $a_{1}^{i} \neq a_{2}^{i}$, from $\Delta_{i}>\left|a_{1}^{i}-a_{2}^{i}\right|$ it is straightforward to see that $a_{j}^{i}-\lambda_{2}^{i}>0, j=1,2$.

We summarize the previous comments in the following result.

Lemma 2.1. Consider the matrix $A$ given by (2). If $\rho(A) \geq 1$ then there is at least $i \in\{1, \cdots, n\}$ such that $d_{1}^{i} d_{2}^{i} \neq 0$

\section{Control design. $N$-periodic feedback approach}

To attenuate the disturbance effect we propose an $N$-periodic approach. Moreover, we make a theoretical treatment of closed-loop stability with a periodic feedback. So, we establish a procedure from a periodic control law in order to ensure that under this control, the closed-loop model remains stable. We also determine the maximum value of the period that allows achieving this goal.

On the system (1) we apply an $N$-periodic control feedback, $N \in \mathbb{Z}, u(k)=$ $-F(k) x(k)$ such that the $N$-periodic closed-loop system is given by

$$
x(k+1)=\hat{A}(k) x(k), \quad \hat{A}(k+N)=\hat{A}(k), k \geq 0,
$$

with $\hat{A}(k)=A-B F(k), k \geq 0$.

It is known that an $N$-periodic system has associated a collection of $N$ invariant systems (see Bittanti (1986); Meyer \& Burrus (1975)), given by

$$
x_{s}(k+1)=\hat{A}_{s} x_{s}(k), k \geq 0, s=0,1, \ldots, N-1,
$$


where $x_{s}(k)=x(k N+s)$ and $\hat{A}_{s}=\Phi_{\hat{A}}(N+s, s)$, being $\Phi_{\hat{A}}\left(k, k_{0}\right)$ defined as

$$
\Phi_{\hat{A}}\left(k, k_{0}\right)=\prod_{i=k_{0}}^{k-1} \hat{A}\left(k_{0}+k-1-i\right), k>k_{0}
$$

and $\Phi_{\hat{A}}\left(k_{0}, k_{0}\right)=I_{n}$. The coefficient matrix $\hat{A}_{s}$ is called the monodromy matrix of time $s$.

It is known (see Bittanti (1986); Bru \& Hernández (1989)) that the non-negativity and asymptotic stability properties are transferred between an $N$-periodic model and its $N$ associated invariant systems. Then, the system (3) is non-negative and asymptotically stable if and only if the monodromy matrix $\hat{A}_{s}=\Phi_{\hat{A}}(N+s, s) \geq 0$ and $\rho\left(\hat{A}_{s}\right)<1, s=0,1, \ldots, N-1$.

From definition of the monodromy matrix, it is clear that if the periodic matrices, $\hat{A}(k), k=0,1, \ldots, N-1$ are non-negative then $\hat{A}_{s}$ is non-negative for $s=0,1, \ldots, N-$ 1. On the other hand, all matrices $\hat{A}_{s}$ have the same spectrum, then we only need to study the case $s=0, \rho\left(\hat{A}_{0}\right)=\rho\left(\Phi_{\hat{A}}(N, 0)\right)<1$ (see, for instance Cantó et al. (2014c)).

Before addressing our study, we recall that a matrix $X \in \mathbb{R}^{q \times p}$ is called an $\{1\}$ inverse of $A \in \mathbb{R}^{p \times q}$ if $A X A=A$. An $\{1\}$-inverse of $A$ is denoted by $A^{(1)}$.The construction of $\{1\}$-inverses for an arbitrary finite matrix $A$ of rank $r>0$ is given in Ben-Israel \& Greville (1974).

The following result is easy to check.

Lemma 3.1. Consider $B \in \mathbb{R}^{n \times m}$ and $M \in \mathbb{R}^{n \times n}$. If there exists some $B^{(1)}$ such that $\left(I-B B^{(1)}\right) M=0$ then the matrix $F=B^{(1)} M$ is a solution of the matrix system $B F=M$.

The main aim of this section is the construction of the $N$-periodic control feedback. This construction is given in the following result.

Lemma 3.2. Consider the system $(A, B)$ given in (1), $M \in \mathbb{R}^{n \times n}$ and $B^{(1)}$ satisfying $\left(I-B B^{(1)}\right) M=0$. If $\rho\left(A^{N-1}(A-M)\right)<1$ for some $N \in \mathbb{Z}$, then there exists an $N$-periodic feedback such that the $N$-periodic closed-loop system (3) is asymptotically stable.

Proof. From Lemma 3.1 we have that $F=B^{(1)} M$ satisfies $B F=M$, then, we consider the $N$-periodic control $u(k)=-F(k) x(k)$ defined as

$$
F(0)=B^{(1)} M, \text { and } F(k)=O, k=1, \ldots, N-1 .
$$

We apply this $N$-periodic control feedback, $u(k)=-F(k) x(k)$ to the system $(A, B)$. Then, we obtain an autonomous periodic closed-loop system where the state matrices are $\hat{A}(k)=A-B F(k), k \geq 0$, where

$$
\hat{A}(0)=A-M \text { and } \hat{A}(k)=A, k=1, \cdots, N-1 .
$$

For studying the asymptotic stability we consider the $N$ invariant systems associated with the periodic system. Since all matrices $\hat{A}_{s}$ have the same spectrum, we look at $\hat{A}_{0}=\Phi_{\hat{A}}(N, 0)=A^{N-1}(A-M)$. So, if $\rho\left(A^{N-1}(A-M)\right)<1$ the collection of $N$ invariant systems will be asymptotically stable and consequently will be the periodic system. 
At this point, the construction of the $N$-periodic control feedback is done using some matrix $M$ made from the structure of the matrix $A$. Denoting $Q_{j}^{l}=\left(\begin{array}{cc}O & O \\ O & I_{j}\end{array}\right) \in \mathbb{R}^{2 j \times 2 j}$ and $Q_{j}^{u}=\left(\begin{array}{cc}I_{j} & O \\ O & O\end{array}\right) \in \mathbb{R}^{2 j \times 2 j}$, we consider two cases to construct this periodic feedback.

Case 1: $M=A Q_{n}^{l}$. In this case, $\hat{A}_{0}=\Phi_{\hat{A}}(N, 0)=A^{N-1}(A-M)=A^{N} Q_{n}^{u}$. The key to solve the stabilization problem is the following theorem.

Theorem 3.3. Consider the unstable system given in (1)-(2) and suppose that $A_{j}, j=$ 1,2 , is stable and $a_{1}^{i} a_{2}^{i}-d_{1}^{i} d_{2}^{i}>0, i=1, \ldots, n$. If for some $B^{(1)},\left(I-B B^{(1)}\right) A Q_{n}^{l}=O$, then there exists an integer $N^{\star}$ such that the $N^{\star}$-periodic control feedback constructed as in (4) provides a non-negative and asymptotically stable closed-loop system (3).

Proof. From Lemma 3.1, there exists a matrix $F$ such that $B F=A Q_{n}^{l}$, then, we define the $N$-periodic control $u(k)=-F(k) x(k)$ as in (4). The $N$-periodic closed-loop system has the state matrices

$$
\hat{A}(0)=A-A Q_{n}^{l}=A Q_{n}^{u} \geq 0 \text { and } \hat{A}(k)=A \geq 0, k=1, \cdots, N-1 .
$$

Then, the $N$-periodic closed-loop system is non-negative and the monodromy matrix at time 0 is $\hat{A}_{0}=A^{N} Q_{n}^{u} \geq 0$. From Lemma 3.2 we have to analyze the spectral radius of the matrix $\hat{A}_{0}$. Note that, using the permutation matrix $P=$ $\left(\begin{array}{lllllllll}e_{1} & e_{3} & \cdots & e_{2 n-1} & e_{2} & e_{4} & \cdots & e_{2 n}\end{array}\right)$, where $e_{i}$ is the $i$ th-unit vector, $i=1, \ldots, 2 n$, we have that $P \hat{A}_{0} P^{T}$ is a lower triangular block matrix with $\operatorname{diag}\left(P \hat{A}_{0} P^{T}\right)=\left(\tilde{A}_{i}^{N} Q_{1}^{u}\right)$. Thus,

$$
\left|\lambda I-\hat{A}_{0}\right|=\prod_{i=1}^{n}\left|\lambda I-\tilde{A}_{i}^{N} Q_{1}^{u}\right|
$$

and we need to study the eigenvalues of matrices $\tilde{A}_{i}^{N} Q_{1}^{u}, i=1,2, \ldots, n$.

Since the system (1) is not stable we know that at least one block $\tilde{A}_{i}$ is not stable, with $\rho\left(\tilde{A}_{i}\right)=\lambda_{1}^{i} \geq 1$. If $\lambda_{1}^{i}=\lambda_{2}^{i}$, from Lemma 2.1 both would be less than one and this contradicts the starting hypothesis, thus $\lambda_{1}^{i} \neq \lambda_{2}^{i}$. Moreover, us$\operatorname{ing} T=\left(\begin{array}{cc}\frac{\lambda_{1}^{i}-a_{2}^{i}}{d_{2}^{i}} & \frac{\lambda_{2}^{i}-a_{2}^{i}}{d_{2}^{i}} \\ 1 & 1\end{array}\right)$, we have that

$$
T \tilde{A}_{i}^{N} Q_{1}^{u} T^{-1}=\left(\begin{array}{cc}
0 & * \\
0 & \rho\left(\tilde{A}_{i}^{N} Q_{1}^{u}\right)
\end{array}\right) \text { with } \rho\left(\tilde{A}_{i}^{N} Q_{1}^{u}\right)=\frac{\lambda_{1}^{i N}\left(\lambda_{1}^{i}-a_{2}^{i}\right)+\left(a_{2}^{i}-\lambda_{2}^{i}\right) \lambda_{2}^{i N}}{\lambda_{i}^{1}-\lambda_{i}^{2}},
$$

and $*$ denotes a suitable scalar. In order to analyze the spectral radius of $\hat{A}_{0}=A^{N} Q_{n}^{u}$, we consider the set of indices $J=\left\{i \in I / \lambda_{1}^{i} \geq 1\right\}$. If $\lambda_{1}^{i}=1$, we have $\rho\left(\tilde{A}_{i}^{N} Q_{1}^{u}\right)=$ $\frac{\left(1-a_{2}^{i}\right)+\left(a_{2}^{i}-\lambda_{2}^{i}\right) \lambda_{2}^{i}}{1-\lambda_{i}^{2}}$. Since $\lambda_{2}^{i}<\lambda_{1}^{i}$, it is easy to prove that $\rho\left(\tilde{A}_{i}^{N} Q_{1}^{u}\right)<1, \forall N>$ 0 . Thus, we focus our attention in the case $\lambda_{1}^{i}>1$. It satisfies $\frac{\lambda_{1}^{i}-\lambda_{2}^{i}}{a_{2}^{i}-\lambda_{2}^{i}}>0$ and we 
want to find a value $N_{i}$ such that

$$
\frac{\lambda_{1}^{i^{N_{i}}}-\lambda_{2}^{i N_{i}}}{\lambda_{1}^{i N_{i}}-1}>\frac{\lambda_{1}^{i}-\lambda_{2}^{i}}{a_{2}^{i}-\lambda_{2}^{i}} .
$$

To guarantee the existence of $N_{i}$, we define a continuous function

$$
g(z)=\frac{\lambda_{1}^{i z}-\lambda_{2}^{i z}}{\lambda_{1}^{i z}-1}
$$

which satisfies $g(1)>\frac{\lambda_{1}^{i}-\lambda_{2}^{i}}{a_{2}^{i}-\lambda_{1}^{i}}$. From $a_{1}, a_{2}<1$ and $a_{1}^{i} a_{2}^{i}-d_{1}^{i} d_{2}^{i}>0$ it follows that $0<\lambda_{2}^{i}<1$. As $\lambda_{2}^{i}<1<\lambda_{1}^{i}$, we have $g(z)>1$ for all $z \geq 1$ and

$$
\lim _{z \rightarrow>\infty} g(z)=\lim _{z->\infty} \frac{1-\left(\frac{\lambda_{2}^{i}}{\lambda_{1}^{i}}\right)^{z}}{1-\left(\frac{1}{\lambda_{1}^{i}}\right)^{z}}=1 .
$$

In addition, the function $g(z)$ is decreasing, for it is enough to prove that $g^{\prime}(z)<0$. The sign of $g^{\prime}(z)$ will be given by the numerator, which in a simplified way, is given by

$$
\left(\lambda_{2}^{i}\right)^{z} \ln \left(\lambda_{2}^{i}\right)-\left(\lambda_{1}^{i}\right)^{z} \ln \left(\lambda_{1}^{i}\right)+\left(\lambda_{1}^{i}\right)^{z}\left(\lambda_{2}^{i}\right)^{z}\left(\ln \left(\lambda_{1}^{i}\right)-\ln \left(\lambda_{2}^{i}\right)\right.
$$

Since $\lambda_{2}^{i}<1$ we have $\left(\lambda_{1}^{i}\right)^{z} \ln \left(\lambda_{1}^{i}\right)<\left(\lambda_{1}^{i}\right)^{z}\left(\lambda_{2}^{i}\right)^{z} \ln \left(\lambda_{1}^{i}\right)$ and since $\lambda_{1}^{i}>1$ we have $\left(\lambda_{2}^{i}\right)^{z} \ln \left(\lambda_{2}^{i}\right)<\left(\lambda_{1}^{i}\right)^{z}\left(\lambda_{2}^{i}\right)^{z} \ln \left(\lambda_{2}^{i}\right)$. Thus,

$$
-\left(\lambda_{2}^{i}\right)^{z} \ln \left(\lambda_{2}^{i}\right)+\left(\lambda_{1}^{i}\right)^{z} \ln \left(\lambda_{1}^{i}\right)>\left(\lambda_{1}^{i}\right)^{z}\left(\lambda_{2}^{i}\right)^{z}\left(\ln \left(\lambda_{1}^{i}\right)-\ln \left(\lambda_{2}^{i}\right),\right.
$$

and this implies that $g^{\prime}(z)<0$. Hence, there is some value $z_{0}$ such that $g(z)>\frac{\lambda_{1}^{i}-\lambda_{2}^{i}}{a_{2}^{i}-\lambda_{1}^{i}}$, for all $z \leq z_{0}$. Thus, it is sufficient to take $N_{i}$ as the integer part of $z_{0}$. Then, from (5) we have that

$$
\begin{aligned}
& \frac{\lambda_{1}^{i}-\lambda_{2}^{i}}{a_{2}^{i}-\lambda_{2}^{i}}\left(\left(\lambda_{1}^{i}\right)^{N_{i}}-1\right)<\left(\lambda_{1}^{i}\right)^{N_{i}}-\left(\lambda_{2}^{i}\right)^{N_{i}}, \\
& \frac{\lambda_{1}^{i}-a_{j}^{i}}{a_{j}^{i}-\lambda_{2}^{i}}\left(\lambda_{1}^{i}\right)^{N_{i}}+\left(\lambda_{2}^{i}\right)^{N_{i}}<\frac{\lambda_{1}^{i}-\lambda_{2}^{i}}{a_{j}^{i}-\lambda_{2}^{i}}, \\
& \left(\lambda_{1}^{i}\right)^{N_{i}}\left(\lambda_{1}^{i}-a_{j}^{i}\right)+\left(\lambda_{2}^{i}\right)^{N_{i}}\left(a_{j}^{i}-\lambda_{2}^{i}\right)<\lambda_{1}^{i}-\lambda_{2}^{i} .
\end{aligned}
$$

So we have obtained $\rho\left(\tilde{A}_{i}^{N_{i}} Q_{1}^{u}\right)<1$. Taking $N^{\star}=\min \left\{N_{i}, i \in J\right\}$ we can ensure that the matrix $\hat{A}_{0}=\Phi_{\hat{A}}\left(N^{\star}, 0\right)$ is stable and the closed-loop system of period $N^{\star}$ is asymptotically stable.

Remark 1. The effect of the feedback control is a disconnection between the two subsystems. We want to delay as much as possible this control action to optimize the costs. Then, we look for the maximum period $N$ such that the $N$-periodic system (3) 
is asymptotically stable, or equivalently, we are interested in the maximum value of $N$ such that $\rho\left(\Phi_{\hat{A}}(N, 0)\right)<1$ and $\rho\left(\Phi_{\hat{A}}(N+1,0)\right)>1$.

Using the function $g(z)$ defined in $(6)$, as $g(z)$ is decreasing we can ensure that there exists $N_{i}$ (the integer part of $z_{0}$ ) such that

$$
g(1)>\cdots>g\left(N_{i}\right)=\frac{\left(\lambda_{2}^{i}\right)^{N_{i}}-\left(\lambda_{1}^{i}\right)^{N_{i}}}{1-\left(\lambda_{1}^{i}\right)^{N_{i}}}>\frac{\lambda_{1}^{i}-\lambda_{2}^{i}}{a_{2}^{i}-\lambda_{2}^{i}}>\frac{\left(\lambda_{2}^{i}\right)^{N_{i}+1}-\left(\lambda_{1}^{i}\right)^{N_{i}+1}}{1-\left(\lambda_{1}^{i}\right)^{N_{i}+1}}=g\left(N_{i}+1\right) .
$$

So, it is sufficient to take $N^{\star}=\min \left\{N_{i}, i \in J\right\}$ as the optimal period which makes the closed-loop system (3) is asymptotically stable. That is, the $N$-periodic system is asymptotically stable if $N \leq N^{\star}$ and it is unstable if the period is $N>N^{\star}$.

We summarize the previous comments on the following result.

Corollary 3.4. $N^{\star}=\min \left\{N_{i}, i \in J\right\}$ is the optimal period which makes the closedloop system (3) asymptotically stable.

Case 2: $M=A Q_{n}^{u}$. In this case, $\hat{A}_{0}=\Phi_{\hat{A}}(N, 0)=A^{N-1}(A-M)=A^{N} Q_{n}^{l}$. The stabilization problem is solved in the following theorem.

Theorem 3.5. Consider the unstable system given in (1)-(2) and suppose that $A_{j}, j=$ 1,2 , is stable and $a_{1}^{i} a_{2}^{i}-d_{1}^{i} d_{2}^{i}>0, i=1, \ldots, n$. If for some $B^{(1)},\left(I-B B^{(1)}\right) A Q_{n}^{u}=O$, then there exists an integer $N^{\star}$ such that the $N$-periodic control feedback constructed as in (4) provides a non-negative and asymptotically stable closed-loop system (3) if $N \leq N^{\star}$ and unstable when $N>N^{\star}$.

Proof. From Lemma 3.1, there exists a matrix $F$ such that $B F=A Q_{n}^{u}$, then, we define the $N$-periodic control $u(k)=-F(k) x(k)$ as (4). To analyze the eigenvalues of the matrix $\hat{A}_{0}$ we observe that, in this case, $\hat{A}_{0}=A^{N} Q_{n}^{l}$. By a simple calculation we can determine that the spectral radius of this matrix can be calculated from the eigenvalues using the following expression

$$
\rho\left(\tilde{A}_{i}^{N} Q_{1}^{l}\right)=\frac{\lambda_{1}^{i N}\left(\lambda_{1}^{i}-a_{1}^{i}\right)+\left(a_{1}^{i}-\lambda_{2}^{i}\right) \lambda_{2}^{i N}}{\lambda_{1}^{i}-\lambda_{2}^{i}}
$$

We can now proceed analogously to the proof of Theorem 3.3, by simply changing $a_{2}^{i}$ by $a_{1}^{i}$, and we obtain the desired result.

Remark 2. It is clear that applying the invariant feedback $u(k)=-F x(k)$ to the initial system is not profitable because that would mean completely decoupling the system. However, with periodic feedback we decouple it only in an instant, allowing the system to stay connected during the remaining steps.

Remark 3. Note that, if $B$ is a non-singular matrix, the equation $B F=A Q_{n}^{u}$ has solution. Moreover, for example, in some biological applications, when the matrices $A_{i}$ represents the transition matrix and $F$ represents the reproduction or infection matrix, the matrix $B=I$. 


\section{Example}

There exists several real processes of different areas where the results obtained in this work can be applied. For instance, a communication networks where the state variable are the hosts files and the links represent the physical connections among them or a social network that reflects friendship relationships among end-users. In both cases the periodic feedback can be interpreted as a disconnection of the system.

The study of the population dynamic in a compartmental model that describes the growth and dispersal of two species among two patches, for example to model a population of idealized insects, can also be addressed using this approach. In this case, feedback is interpreted as the application of control techniques to avoid or to minimize the pest's impact on the patches by applying a systemic insecticide on the patch to prevent insect infestations or by tacking sanitation measures, such as cleaning up and destroying any materials that come from trees or shrubs that are already infested.

So, the result given in Corollary 3.4, $N_{0}$ will represent the number of stages we can perform without disconnecting or without applying the control so that the process is stable. Moreover, although it is possible to use constant feedback, that would not be interesting because it means that the control action is applied at all times. In the cases that we propose we are interested in delaying that control action to the maximum. In the next subsection we develop the third application and illustrate the procedure that allows us to obtain the maximum number of steps to determine the period of feedback.

\subsection{Numerical example}

To illustrate the applicability of the results we consider a coupled compartmental systems that describes the growth and dispersal of two species of insects among two patches. To simplify the example we include all the individuals in two stages: immature stage and adult stage. The state variable depends on the age of the individual and it is into account the existence of migration between patches. The state vector is given by $x(\cdot)=\left(\begin{array}{l}x_{1}(\cdot) \\ x_{2}(\cdot)\end{array}\right)$, with $x_{i}(\cdot)=\left(\begin{array}{l}x_{1 i}(\cdot) \\ x_{2 i}(\cdot)\end{array}\right) \geq 0, i=1,2$, such that $x_{i j}(\cdot)$ denotes population density of species in the stage $i$ of species $j$ at time $t$, and taking $u(\cdot)=\left(u_{i}(\cdot)\right)_{i=1}^{4} \geq 0$ as a control vector, the dynamic process is represented by

$$
\begin{aligned}
& x_{11}(k+1)=\left(a_{11}+\alpha_{1}\right) x_{11}(k)+d_{112} x_{12}(k)+u_{1}(k) \\
& x_{21}(k+1)=a_{21} x_{21}(k)+b_{11} x_{11}(k)+d_{212} x_{22}(k)+u_{2}(k) \\
& x_{12}(k+1)=\left(a_{12}+\alpha_{2}\right) x_{12}(k)+d_{121} x_{11}(k)+u_{3}(k) \\
& x_{22}(k+1)=a_{22} x_{22}(k)+b_{12} x_{12}(k)+d_{221} x_{21}(k)+u_{4}(k),
\end{aligned}
$$

where $a_{i, j}$ is the rate at which each stage $i$ of species settles on the patch $j, b_{1, i}$ is the rate individuals of species $i$ changing of age-stage, $d_{k i j}$ is the dispersal rate from patch $j$ to patch $i$ of species $k$ and $\alpha_{i}$ represents the rate of replenishment of the species $i$.

In our model, $x(k+1)=A x(k)+B u(k)$, the state coefficient matrix is a stagestructured population matrix and $B=I$. For the purpose of generating a numerical example, we consider the following state matrix satisfying all the hypotheses of the 


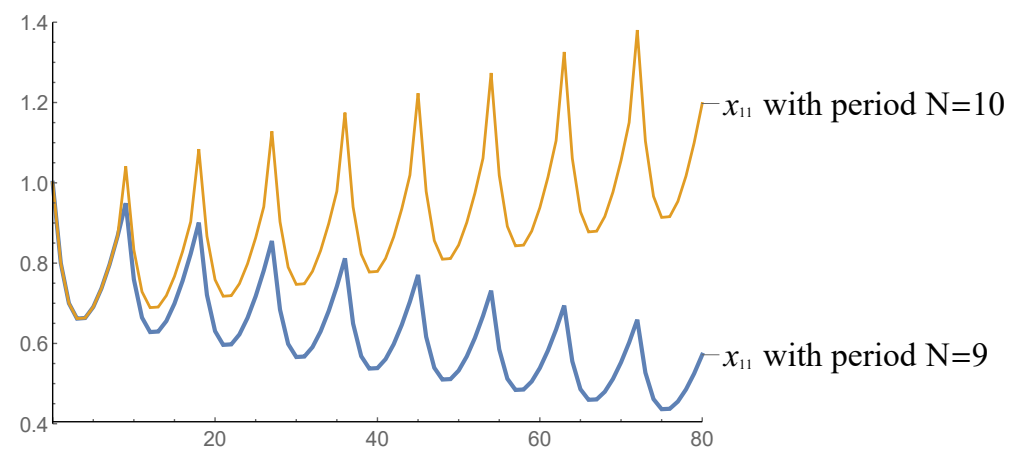

Figure 1. Evolution of the state of the fist compartment using an $N$-periodic control feedback.

Theorem 3.3:

$$
A=\left(\begin{array}{ll}
A_{1} & D_{1} \\
D_{2} & A_{2}
\end{array}\right)=\left(\begin{array}{cc|cc}
0.8 & 0 & 0.1 & 0 \\
0.2 & 0.3 & 0 & 0.03 \\
\hline 0.6 & 0 & 0.9 & 0 \\
0 & 0.7 & 0.2 & 0.97
\end{array}\right)
$$

The matrices $A_{1}$ and $A_{2}$ are stable but $A$ is unstable. We construct the $N$-periodic feedback $u(k)=-F(k) x(k)$ as in (4) where $F=A Q_{2}^{l}$.

This model predicts the number of individuals in each stage through time. The application of the periodic feedback to system may have important effects on persistence and extinction of one population or both populations and the correct method of application is very important to get the best results.

Following the steps given in the theorem we obtain an optimal value for the period. So, for $N^{\star}=9$ the closed-loop system is asymptotically stable since $\hat{A}_{0}=\Phi_{\hat{A}}(9,0)$ has $\rho\left(\hat{A}_{0}\right)=0.949$ but if the period is $N=10$, then the matrix is not stable, since in this case $\rho\left(\hat{A}_{0}\right)=\rho\left(\Phi_{\hat{A}}(5,0)\right)=1.04$. The behaviour of the solution is showed in the Figure 1.

\section{Conclusions}

We consider a compartmental system where each subsystem has communication with the other subsystem. Using a feedback control, better performance can be achieved to stabilize the closed-loop system. That performance improvement is due to the special structure of the model. In addition, the proposed control strategy determines the maximum number of stages in which the closed-loop system remains stable. This information can be crucial when designing different control strategies in real processes of very different fields: electrical engineering, electronic engineering, chemistry, biology or medical engineering, where a periodic type of performance is required.

\section{Acknowledgement}

This research was partially supported by Spanish Grant MTM2013-43678-P. 


\section{References}

Álvarez, J., Pasamontes, M., Guzman, J., \& Camacho, E. (2016). A practical hybrid predictive control algorithm for a low-temperature thermosolar plant. Optimal Control App. and Methods, 37(3), 508-520.

Ahn, H.S., Moore, K.L., \& Chen, Y. (2010). Trajectory-keeping in satellite formation flying via robust periodic learning control. Int. Journal of Robust and Nonlinear Control, 20(14), $1655-1666$.

Berman, A., \& Plemmons, R.J. (1994). Nonnegative Matrices in the Mathematical Sciences. Philadelphia, SIAM.

Ben-Israel, A., \& Greville, T.N.E. (1974). Generalized Inverses: Theory and Applications. New York, Wiley and Sons.

Bhatia, R. (1997). Matrix Analysis. New York, Springer-Verlag.

Bittanti, S. (1986). Deterministic and stochastic linear periodic systems, In: S. Bittanti (Ed.), Time series and linear systems, 141-182. Berlin, Springer-Verlag.

Boccaletti, S., Latora, V., Moreno, Y., Chaves, M. , \& Hwang, D.H. (2006). Complex networks: Structure and dynamics. Physics Reports, 424, 175-308.

Bru, R. \& Hernández, V. (1989). Structural properties of discrete-time linear positive periodic systems. Linear Algebra and its Applications, 121, 171-183.

Cantó, B., Cardona, S.C., Coll, C., Navarro-Laboulais, J. , \& Sánchez, E. (2014a). Dynamic optimization of a gas-liquid reactor. Journal of Mathematical Chemistry , 50 (2), 381-393.

Cantó, B., Coll, C., \& Sánchez, E. (2014b). Structured parametric epidemic models. Int. Journal of Computer Mathematics , 91(2), 188-197.

Cantó, B., Coll, C., \& Sánchez, E. (2014c). A study on vaccination models for a seasonal epidemic process. Applied Mathematics and Computation, 243, 152-160.

Ellis, M., Durand, H., \& Christofides, P.D. (2014). A tutorial review of economic model predictive control methods. Journal of Process Control, 24 (8), 1156-1178.

Li, M.Y., \& Shuai, Z. (2010). Global-stability problem for coupled systems of differential equations on networks. Journal of Differential Equations, 248, 1-20.

Mahmoud, M.S. \& Qureshi, A. (2012). Decentralized sliding-mode output-feedback control of interconnected discrete-delay systems. Automatica, 48, 808-814.

Meyer, R. \& Burrus, C.S. (1975). A unified analysis of multirate and periodically time-varying digital filters. IEEE Transactions on Circuits and Systems, 22 (3), 162-168.

Su, H., Li, W., \& Wang, K. (2012). Global stability analysis of discrete-time coupled systems on networks and its applications. Chaos, 22, 33-35, doi: 10.1063/1.4748851.

Tadeo, F. \& Rami, M.A. (2010). Selection of time-after-injection in bone scanning using compartmental observers. In Proceedings of the Wold Congress on Engineering, WCE 201016 , London,U.K.

Van den Hof, J.M. (1998). Structural identifiability of linear compartmental systems. IEEE Transactions on Automatic Control, 43, 800-818.

Zhang, Y., Wen, C., Soh, Y.C. (2000). Robust decentralized adaptive stabilization of interconnected systems with guaranteed transient performance, Automatica, 36, 907-915. 